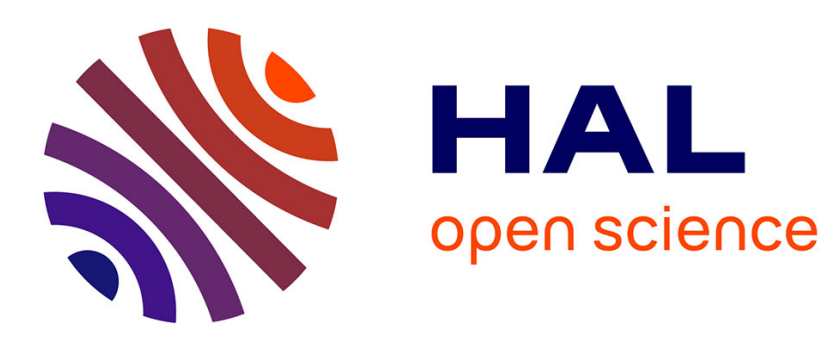

\title{
Phonon energy in an anharmonic quasi-one-dimensional solid
}

\author{
M.A. Grado-Caffaro, M. Grado-Caffaro
}

\section{To cite this version:}

M.A. Grado-Caffaro, M. Grado-Caffaro. Phonon energy in an anharmonic quasi-one-dimensional solid. Journal of Physics and Chemistry of Solids, 2011, 72 (8), pp.957. 10.1016/j.jpcs.2011.05.002 . hal-00768587

\section{HAL Id: hal-00768587 \\ https://hal.science/hal-00768587}

Submitted on 22 Dec 2012

HAL is a multi-disciplinary open access archive for the deposit and dissemination of scientific research documents, whether they are published or not. The documents may come from teaching and research institutions in France or abroad, or from public or private research centers.
L'archive ouverte pluridisciplinaire HAL, est destinée au dépôt et à la diffusion de documents scientifiques de niveau recherche, publiés ou non, émanant des établissements d'enseignement et de recherche français ou étrangers, des laboratoires publics ou privés. 


\section{Author's Accepted Manuscript}

Phonon energy in an anharmonic quasi-onedimensional solid

M.A. Grado-Caffaro, M. Grado-Caffaro

PII:

S0022-3697(11)00130-2

DOI: doi:10.1016/j.jpcs.2011.05.002

Reference: PCS 6459

To appear in:

Journal of Physics and

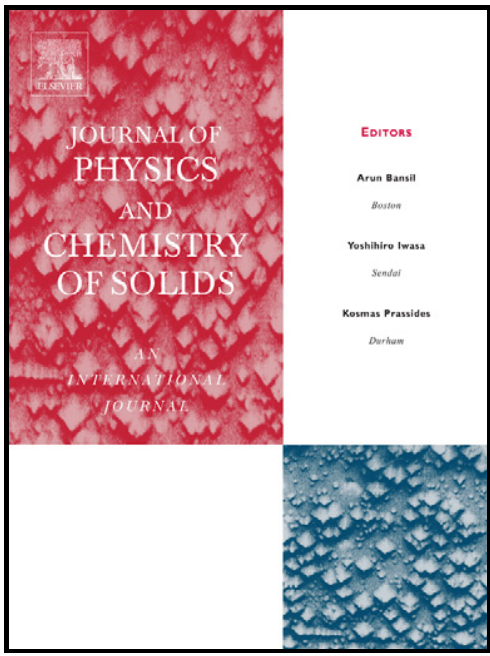

www.elsevier.com/locate/jpcs Chemistry of Solids

Received date: $\quad 21$ January 2011

Revised date: $\quad 2$ April 2011

Accepted date: 6 May 2011

Cite this article as: M.A. Grado-Caffaro and M. Grado-Caffaro, Phonon energy in an anharmonic quasi-one-dimensional solid, Journal of Physics and Chemistry of Solids, doi:10.1016/j.jpcs.2011.05.002

This is a PDF file of an unedited manuscript that has been accepted for publication. As a service to our customers we are providing this early version of the manuscript. The manuscript will undergo copyediting, typesetting, and review of the resulting galley proof before it is published in its final citable form. Please note that during the production process errors may be discovered which could affect the content, and all legal disclaimers that apply to the journal pertain. 
PHONON ENERGY IN AN ANHARMONIC QUASI-ONE-DIMENSIONAL SOLID

By M.A. Grado-Caffaro and M. Grado-Caffaro

Permanent address: M.A. Grado-Caffaro and M. Grado-Caffaro- Scientific Consultants, C/ Julio Palacios 11, 9-B, 28029-Madrid (Spain); www.sapienzastudies.com; e-mail address: ma.grado-caffaro@sapienzastudies.com

Abstract.- For the first time, the phonon energy per unit volume in a large anharmonic quasi-one-dimensional solid is determined by considering all polarizations of the various modes of phonon propagation and by assuming the solid as a lattice of atoms behaving as Morse oscillators. In this context, the equilibrium phonon occupation number, which is given by the Bose distribution, replaces formally the vibrational quantum number into the expression for the Morse-oscillator energy. In addition, the quasi-harmonic solid is discussed within the above framework so that the phonon energy per unit volume is calculated for a large quasi-harmonic and quasi-onedimensional solid.

Key words: D. Phonon energy; D. Anharmonic solid; D. Morse oscillator; D. Bose distribution; D. Quasi-harmonic solid

1. Introduction.- Anharmonic solids appear as very relevant entities in the context of non-conventional solid-state physics. In this respect, building a complete and accurate theory to characterize an anharmonic solid is not certainly an easy task. Work related 
directly or indirectly to the subject as, for example, refs.[1-11] has been done but a lot of questions remain open. In order to fix the main points in relation to describe anharmonicity in the physics of solid state, first we will remember what is a harmonic solid and related issues. A solid is denominated harmonic if the atoms of the corresponding crystalline or distorted lattice behave as quantum harmonic oscillators, that is, the above atoms vibrate harmonically around their respective equilibrium positions. The displacement of an atom from its equilibrium position gives rise to the propagation of vibrational modes in the lattice, these modes carrying energy which is quantized so that every vibrational quantum is called a phonon. A phonon is conceived as a quasi-particle and may be viewed as a packet of travelling waves with a certain frequency, a certain group velocity and involving an energy which equals the Planck constant multiplied by the frequency (see, for instance, refs.[12-16]).

It is well-known that phonons play a notorious role in the physics of condensed matter in conjunction with other elementary excitations as, for example, excitons and polarons. We may consider electron-phonon scattering and phonon-phonon scattering as very relevant processes. In particular, phonon-phonon scattering is an anharmonic mechanism of notable importance. Other significant processes are phonon-assisted optical transitions where, for instance, problems relative to wave-vector conservation acquire a special relevance. On the other hand, note that there are four types of phonon polarization namely: both longitudinal and transversal acoustic phonons as well as both longitudinal and transversal optical ones. In this respect, it is well-known that only acoustic phonons contribute to thermal processes contrary to electronic and optical processes in which only optical phonons contribute [12-16].

With respect to harmonicity or anharmonicity, that is, linearity or nonlinearity, it is clear that assuming anharmonic solids is a framework more realistic and powerful than considering only harmonicity [1-11]. Anharmonicity is a very relevant subject in the context of new and complex materials whose behaviour involves great departures from the conventional, simple models of solid-state physics. Even for simple solids, anharmonicity becomes important for large deviations of the atoms from their respective equilibrium positions. Moreover, the anharmonic character of the atomic motion in solids may bring relevant information to the classical theory of elasticity. Within this context, the Morse potential is a very suitable potential since it allows us to 
envisage corrections to the harmonic oscillations. On the other hand, it is well-known that the so-called Einstein's oscillator model is not realistic (see, for example, refs.[111]). The limitations of the above model are certainly well-known; considering, for instance, the zero-point vibrational energy (which is well defined only when the crystalline solid may be conceived within the harmonic approximation [5,11]), then the Einstein's model does not provide the exact value of the aforementioned energy even in a fully harmonic classical crystal [11]. As a matter of fact, the effective potentials in real crystals exhibit appreciable anharmonic contributions whose evaluation is really hard (see, for instance, ref.[11]). At this point, let us regard quantum crystals as, for example, solid hydrogen, in which anharmonic effects are important; really, these quantum lattices are strongly anharmonic [6,11]. At any rate, in practice, perhaps the notion of quasi-harmonic solid is more realistic and, consequently, more fruitful than the purely anharmonic solid since the atoms of the major part of crystalline or amorphous solids behave as quantum quasi-harmonic oscillators, that is, the above atoms vibrate almost harmonically around their respective equilibrium positions. The fundamental vibrational quanta are called quasi-harmonic phonons (see, for instance, refs.[1,2]). The aim of the present article is, on the one hand, determining the phonon energy per unit volume in a large anharmonic quasi-one-dimensional solid as an arrangement of atoms behaving as Morse oscillators and, on the other hand, finding approximate formulas for the quasiharmonic phonon energy per unit volume at both lower and higher temperatures. In this context, the phonon density of states plays a key role which will be examined within the quasi-harmonic approximation. The paper is organized as follows: section 2 is dedicated to calculate the anharmonic phonon energy per unit volume; section 3 deals with determining the phonon energy per unit volume in a quasi-harmonic solid; section 4 is a discussion of results; concluding remarks are given in section 5.

2. Calculation of the phonon energy per unit volume in an anharmonic solid.- First of all, we consider the equilibrium phonon occupation number, which is given by the Bose distribution namely:

$$
\langle\hat{n} \omega\rangle=\frac{1}{\exp \left(\frac{\hbar \omega}{k_{B} T}\right)-1}
$$


where the aforementioned occupation number is the expectation value of the particlenumber operator $\hat{n} \equiv \hat{A}^{+} \hat{A} \quad\left(\hat{A}^{+}\right.$and $\hat{A}$ being the corresponding creation and annihilation operators, respectively), $\hbar$ is the reduced Planck constant, $\omega$ denotes phonon angular frequency, $k_{B}$ is the Boltzmann constant, and $T$ stands for temperature which, for a given medium under thermodynamic equilibrium, is a well defined quantity.

On the other side, we wish to determine the phonon energy per unit volume assuming a, say, typical anharmonic quasi-one-dimensional solid as a lattice under an interatomic Morse potential. At this point, we want to remark that, obviously, although anharmonicity can be described by a number of potential types, the Morse approach is the most realistic model in the physics of anharmonic solids since the aforementioned approach is an extension of the harmonic-oscillator model as we appreciate when we consider the following expression for the quantized energy of a single-particle Morse oscillator $[3,4,17]$ :

$$
E_{n}=\hbar \omega\left(n+\frac{1}{2}-\varepsilon n^{2}-\varepsilon n-\frac{\varepsilon}{4}\right)
$$

where $n=0,1,2, \ldots$ and $\varepsilon$ is a relatively small anharmonic coefficient (of course, $\varepsilon$ is positive such that $\varepsilon=\hbar \omega / 4 D^{-}$where $D$ is the potential-well depth). Note that $\varepsilon$ is a parameter whose role is corrective with respect to the quantum harmonic oscillator so that, by setting $\varepsilon=0$ into eq.(2), one gets the energy of a one-particle quantum harmonic oscillator. This fact arises from that the Morse potential energy of a given particle, when its position is sufficiently near the equilibrium position, is approximately equal to the potential energy of the particle as harmonic oscillator as we will show as follows. The Morse potential energy reads:

$$
V \mathbb{C}_{-}^{-}=D \mathrm{r}-\exp \mid a \boldsymbol{c}-x_{e} \underset{-2}{-2}
$$

where $x_{e}$ is the position of equilibrium and $a$ is a parameter which controls the potential width. Then, when $x \approx x_{e}$, the first-order Taylor expansion of (3) around $x=x_{e}$, given that $2 D a^{2}=m \omega^{2}$, yields $V \varangle \approx m \omega^{2} \varangle-x_{e}^{2}-2$. On the other hand, note the well-known fact that the Morse potential leads to a finite number of bound 
states; in this respect, see formula (2) or perhaps the more manageable following expression:

$$
E_{n}=\hbar \omega\left(n+\frac{1}{2}\right)\left[1-\frac{\hbar \omega}{4 D}\left(n+\frac{1}{2}\right)\right]
$$

so, by considering relations (2) or (4), we have that $n_{\max } \approx 2 D / \omega \omega \xi 1 / \varepsilon_{\text {. }}^{-}$.

We consider an anharmonic solid whose atoms behave as Morse oscillators. In order to determine the total phonon energy per unit volume, we take into account the contribution from all the possible polarizations of the various modes of phonon propagation namely longitudinal and transverse acoustic as well as longitudinal and transverse optical. This gives a discrete summation over an index that will be denoted by $p$ (polarization). There is another discrete summation over the wave-vector range which is the first Brillouin zone. However, we will assume the solid as sufficiently large which implies that the wave-vector space is very dense so it can be regarded as continuous which permits that this last contribution can be approximated as a definite integral over the wave-vector space and, since the first Brillouin zone is assumed to be isotropic, then integration over the wave-vector space becomes integration over the angular-frequency space [12-16]. Then, substituting formally $n$ into eq.(2) by $\langle\hat{n} \uparrow$, under the above approximation, the energy (per unit volume) in question reads:

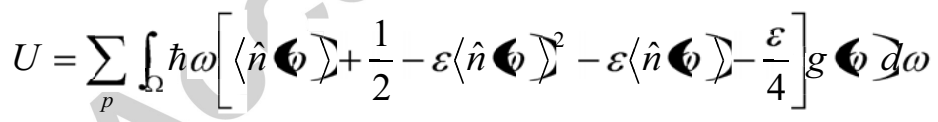

where $g$ denotes phonon density of states and $\Omega$ is the range of phonon angular frequency; this range corresponds, in the wave-vector space, to the first Brillouin zone (note the wave-vector dependence of $\omega$ ).

Now we are interested in determining an approximate expression for the equilibrium phonon occupation number at relatively low temperature, that is, when $k_{B} T<<\hbar$. Under this condition, it is clear that formula (1) becomes: 


$$
\langle\hat{n} \circlearrowleft\rangle_{T \approx 0} \approx \exp \left(-\frac{\hbar \omega}{k_{B} T}\right)
$$

We are also interested in finding another approximate relationship for relatively high temperature, that is, when $k_{B} T \gg \hbar \omega$. This condition, by approximating the exponential in (1) with a first-order Taylor expansion around the origin, reduces (1) to:

$$
\langle\hat{n} \omega\rfloor_{T \rightarrow \infty} \approx \frac{k_{B} T}{\hbar \omega}
$$

Note that expanding the right-hand side of (1) is not possible because this $\omega$-dependent quantity is discontinuous at $\omega=0$.

Then, knowing $g$, and replacing formula (6) (formula (7)) into (5), it is feasible, in principle, to obtain an approximate relation for $U$ at lower (higher) temperatures.

3. Determination of the quasi-harmonic phonon energy per unit volume.- Our purpose is now determining the phonon energy per unit volume within the quasi-harmonic approximation. In other words, we wish to calculate the energy per unit volume due to quasi-harmonic phonons. To get this end, we begin by considering the following expression for the phonon density of states in a quasi-harmonic solid (see, for instance, refs.[18,19]):

$$
g \omega_{0} \approx \frac{1}{N} \sum_{v=1}^{N}\left|a_{v}\right|^{2} \delta\left(v-\omega_{v}\right.
$$

where $a_{v}$ is the matrix element of the oscillator strength, $\omega_{v}$ denotes the $v^{\text {th }}$ eigenfrequency corresponding to every normal mode of the solid, $\delta$ designates Dirac's delta function, and $N$ is the number of atoms of the solid; given that the solid is assumed to be quasi-one-dimensional, $N$ is approximately equal to the number of normal vibrational modes of the solid.

On the other hand, one has to find an approximate formula from the second factor of the integrand in (5) for sufficiently small $\varepsilon$ (quasi-harmonic approximation). To get this 
end, neglecting $\varepsilon / 4$, the factor in question except $1 / 2$ becomes

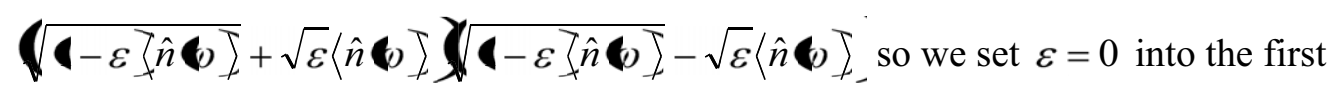

term of the first factor and into the second factor of this last expression which reduces to $\langle\hat{n}\rceil\left(+\sqrt{\varepsilon\left\langle\hat{n}{ }_{0}\right.}\right.$; . This scheme can be viewed as superharmonic approximation; by contrast, setting $\varepsilon=0$ into the second factor (or only into the first term of this factor) and into the first factor, one gets an expression proportional to $\langle\hat{n} \downarrow\rfloor$. In order to gain accuracy, we will employ the superharmonic approximation. On this basis and replacing relationships (6) and (8) into (5), we obtain, at low temperature:

$U \approx 0 \approx \frac{\hbar}{N} \sum_{p} \sum_{v=1}^{N}\left|a_{v}\right|^{2} \omega_{v}\left[\frac{1}{2}+\exp \left(-\frac{\hbar \omega_{v}}{k_{B} T}\right)+\sqrt{\varepsilon} \exp \left(-\frac{3 \hbar \omega_{v}}{2 k_{B} T}\right)\right]$

and, at high temperature, under the approximation in question and substituting (7) and (8) into (5), it follows:

$U \boldsymbol{C} \rightarrow \infty \approx \frac{1}{N} \sum_{p} \sum_{v=1}^{N}\left|a_{v}\right|^{2}\left(\frac{1}{2} \hbar \omega_{v}+k_{B} T+\frac{k_{B}^{3 / 2} T^{3 / 2} \sqrt{\varepsilon}}{\sqrt{\hbar \omega_{v}}}\right)$

For the sake of brevity of notation and since, in practice, only one type of polarization takes place, from now on we will omit the summation symbol relative to polarization.

When $N \rightarrow \infty$ (large solid), by using Stolz's theorem, expressions (9) and (10) become respectively:

$U \boldsymbol{C} \approx 0 ; N \rightarrow \infty \approx \hbar \lim _{N \rightarrow \infty}\left\{\left|a_{N}\right|^{2} \omega_{N}\left[\frac{1}{2}+\exp \left(-\frac{\hbar \omega_{N}}{k_{B} T}\right)+\sqrt{\varepsilon} \exp \left(-\frac{3 \hbar \omega_{N}}{2 k_{B} T}\right)\right]\right\}$

$U \rightarrow \infty ; N \rightarrow \infty \approx \lim _{N \rightarrow \infty}\left[\left|a_{N}\right|^{2}\left(\frac{1}{2} \hbar \omega_{N}+k_{B} T+\frac{k_{B}^{3 / 2} T^{3 / 2} \sqrt{\varepsilon}}{\sqrt{\hbar \omega_{N}}}\right)\right]$ 
From formula (11) it follows:

$$
U \boldsymbol{\top}=0 ;\left.N \rightarrow \infty \approx \frac{\hbar}{2} \lim _{N \rightarrow \infty} \boldsymbol{( a}_{N}\right|^{2} \omega_{N}
$$

On the other hand, since typically $\varepsilon \approx 0.1$ and $k_{B} T \approx 10 \hbar \omega_{N}$, from relation (12) we get:

$$
U \rightarrow \infty ; N \rightarrow \infty \approx 2 k_{B} T \lim _{N \rightarrow \infty}\left|a_{N}\right|^{2}
$$

4. Discussion.- In the spirit of taking into account anharmonic effects, we have treated the determination of the phonon energy per unit volume in a large anharmonic quasione-dimensional solid as a crystalline or distorted lattice in which the atoms vibrate as Morse oscillators around their respective equilibrium positions. Moreover, the phonon energy per unit volume has been calculated for a large quasi-harmonic quasi-onedimensional solid. In order to calculate the energy density in question, we have taken into consideration the four possible modes of phonon propagation namely: longitudinal and transversal acoustical as well as longitudinal and transversal optical. In this respect, we want to remark the following well-known facts: the contribution of optical phonons to thermal transport is certainly small and the contribution of acoustical phonons to electronic transport is also small. In order to study electronic transport and the optical properties of an anharmonic solid or a quasi-harmonic one, it is evident that only optical phonons must be considered. In this context, we can mention, as a notorious example, optical absorption in both crystalline and amorphous solids. For instance, the coefficient of infrared absorption in an amorphous solid due to structural disorder reads $\alpha \omega_{-}=\mu I^{2} g \omega_{\text {, where }} \mu \omega_{-}$is the dipole-moment matrix element in the angular-frequency domain, $\eta$ stands for refractive index, and $c$ is the speed of light in vacuum (see, for example, ref.[20]) while the total coefficient of infrared absorption is $\alpha \omega=\mid \mu\rfloor^{2} g * g \backslash \alpha c \omega_{-}^{-}$where the asterisk denotes convolution (see, for instance, ref.[21]). Therefore, the coefficient of infrared absorption relative to dynamical disorder reads $\alpha$ mention that this coefficient depends only on transverse optical phonons in amorphous 
III-V compounds [20]. From the above-mentioned relationship for $\alpha$, one has that $g=\eta c \omega \alpha{ }_{\mathcal{L}}=\mathrm{I}^{2}$ which, inserted into (5) and taking into account eq.(1), allows the evaluation of $U$ when we know $\alpha$

5. Concluding remarks.- Integrating both sides of relationship (8) over a suitable angular-frequency range and by using Stolz's theorem, one has that $\widetilde{N}=\lim _{N \rightarrow \infty}\left|a_{N}\right|^{2}$ where $\widetilde{N}$ is the number of phonon states in the above range for a sufficiently large solid. In terms of this number of states, formulas (13) and (14) become respectively $U \boldsymbol{C}=0 ; N \rightarrow \infty \approx \frac{\hbar \tilde{N}}{2} \lim _{N \rightarrow \infty} \omega_{N}, U \boldsymbol{C} \rightarrow \infty ; N \rightarrow \infty \approx 2 k_{B} T \tilde{N}$. Therefore, from these expressions, we infer that $U$ (at zero $T$ and also at sufficiently high $T$ ) is approximately proportional to the number of phonon states in a large enough solid. In contrast, when $N$ is relatively small, the above two expressions are obviously not applicable so that we can use formulae (9) and (10), which refer to finite $N$. This situation presents interest in elucidating diverse mechanisms related to elementary excitations in nanosystems [22,23].

\section{REFERENCES}

[1] T. Yildirim, Solid State Commun. 124 (2002) 449-455.

[2] S. Jiuxun, Y. Hongchun, W. Qiang, C. Lingcang, J. Phys. Chem. Solids 63 (2002) 113-117.

[3] M.A. Grado-Caffaro, M. Grado-Caffaro, Eur. Phys. J. B 73 (2010) 243-245.

[4] M.A. Grado-Caffaro, M. Grado-Caffaro, Physica B 406 (2011) 231-233.

[5] M. Born, K. Huang: Dynamical Theory of Crystal Lattices (Clarendon Press, Oxford, 1996). 
[6] M.L. Klein, J.A. Venables: Rare Gas Solids, Vol.1 (Academic Press, New York, 1970).

[7] P. Diep, J.K. Johnson, J. Chem. Phys. 112 (2000) 4465.

[8] R.J. Hinde, J. Chem. Phys. 128 (2008) 154308.

[9] R.J. Hinde, Chem. Phys. Lett. 460 (2008) 141.

[10] K. Rosciszewski, B. Paulus, P. Fulde, H. Stoll, Phys. Rev. B 62 (2000) 5482.

[11] K. Rosciszewski, B. Paulus, Molecular Phys. 108 (2010) 2147-2152.

[12] C. Kittel: Introduction to Solid State Physics (John Wiley \& Sons, Inc., New York, N.Y., Sixth Edition, 1986).

[13] C. Kittel: Quantum Theory of Solids (John Wiley \& Sons, Inc., New York, N.Y., Second Edition, revised, 1987).

[14] C.L. Tien, A. Majumdar, F.M. Gerner (Eds.): Microscale energy transport (Taylor and Francis, 1998).

[15] Y.S. Ju, K.E. Goodson, Appl. Phys. Lett. 74 (1999) 3005-3007.

[16] S. Mazumder, A. Majumdar, J. Heat Transfer 123 (2001) 749-759.

[17] J.J.-L. Ting, J. Phys. B: At. Mol. Opt. Phys. 11 (2008) 1-8.

[18] J.D. Axe, D.T. Keating, G.S. Cargill III, R. Alben, AIP Conf. Proc. 20 (1974) 279.

[19] M.A. Grado-Caffaro, M. Grado-Caffaro, Mod. Phys. Lett. B 18 (2004) 1255-1259.

[20] M.A. Grado-Caffaro, M. Grado-Caffaro, Phys. Lett. A 169 (1992) 399-401. 
[21] H.K. Rockstad, AIP Conf. Proc. 20 (1974) 21.

[22] V. Ermakov, S. Kruchinin, H. Hori, A. Fujiwara, Int. J. Mod. Phys. B 21 (2007) 1827-1835.

[23] A. Soldatov, N. Bogolubov, Jr., S. Kruchinin, Condens. Matter Phys. 9 (2006) 1-9. 
explanation is supported sufficiently by refs.[1-4] (already mentioned in the former version) and 7 additional references. Consequently, the revised version of the paper is manifestly accessible to the readers so it satisfies clearly the questions formulated by reviewer 3 . 
PHONON ENERGY IN AN ANHARMONIC QUASI-ONE-DIMENSIONAL SOLID

By M.A. Grado-Caffaro and M. Grado-Caffaro

\section{RESEARCH HIGHLIGHTS}

1. Phonon energy of an anharmonic solid is found.

2. Atoms are assumed as Morse oscillators.

3. Quasi-harmonic phonon energy is also found.

4. All phonon propagation modes are considered. 